\title{
Low Phase Noise Direct-Modulation Optoelectronic Oscillator
}

\author{
Brian Sinquin, Marco Romanelli, Steve Bouhier, \\ Ludovic Frein, Mehdi Alouini, Marc Vallet \\ Institut FOTON, UMR 6082, Univ. Rennes1 \\ brian.sinquin@univ-rennes1.fr
}

September 13, 2021

\begin{abstract}
A direct-modulation OEO (DM-OEO) generating stable $10 \mathrm{GHz}$ and $20 \mathrm{GHz}$ signals is presented. A single loop and a dual loop approach are implemented and compared. We show an output signal of $15 \mathrm{dBm} R F$ power, and a phase noise as low as -135 $\mathrm{dBc} / \mathrm{Hz}$ at $10 \mathrm{kHz}$ offset from the $10 \mathrm{GHz}$ carrier. The $20 \mathrm{GHz}$ second harmonic exhibits a noise level of $-127 \mathrm{dBc} / \mathrm{Hz}$ at $10 \mathrm{kHz}$. A high spur level reduction is also obtained in the dual loop architecture.
\end{abstract}

\section{INTRODUCTION}

The generation of pure microwave signals with low phase noise is a requirement for applications as diverse as coherent telecommunications, clock sharing, or tele-detection. Methods based on quartz oscillators and frequency multiplication for generating high frequency carriers are not sufficient for current needs, and a new generation scheme called Opto-Electronic Oscillator (OEO) was introduced in the late 90's to lower the phase noise at high carrier frequencies (typically a few tens of $\mathrm{GHz}$ ) [1]. This system is based on the insertion of a low loss $(0.2 \mathrm{~dB} / \mathrm{km}) \mathrm{km}$-long optical fiber in an opto-RF feedback loop. The optical part of the loop serves as a long delay line, resulting in a high-Q resonator for the optically-carried RF signal. Most of the OEO architectures include a Mach-Zehnder Modulator (MZM) as an external optical intensity modulation element [2]. An alternative to this widespread scheme is direct modulation (DM) of the injection current of a semiconductor laser. DM is arguably the simplest possible OEO scheme [3], and offers a cheaper and more compact approach. In comparison with the huge literature on the classical MZM-based architecture, the DM approach has been much less explored. An early paper studying the effect of delayed feedback on the laser current is [4]. At that time people were strongly interested in instabilities, however the authors already noted that such a system "may be conveniently used as a high frequency source". Many subsequent studies on semiconductor lasers with optoelectronic feedback were indeed focused on dynamical instabilities [2]. More recently, some authors proposed setups based on various combinations of optoelectronic feedback and optical injection [5] or feedback [6], [7] with the aim of generating stable and possibly tunable microwave signals. Direct current modulation of a microsquare laser was used in [8] to generate tunable signals with good phase noise. Very recently, an architecture similar to the one presented here was published [9], showing OEO operation between 8 and $12 \mathrm{GHz}$.

In this paper, we implement the DM-OEO scheme in its simplest form, and study in detail its performances as a generator of high frequency signals. In addition to the single-loop architecture, we study a dual-loop scheme which improves some figures of the $\mathrm{OEO}$, at the expense of an increased complexity. Our system exhibits stable single-frequency oscillation of a $10 \mathrm{GHz}$ carrier, with $15 \mathrm{dBm}$ output RF power and $-135 \mathrm{dBc} / \mathrm{Hz}$ phase noise at $10 \mathrm{kHz}$ offset from the carrier. The rejection ratio of adjacent resonant modes is $60 \mathrm{~dB}$ in the single-loop scheme, and more than 80 $\mathrm{dB}$ with the dual loop. Furthermore, due to nonlinearities in the feedback loop, $20 \mathrm{GHz}$ output is also available. These results constitute, to the best of our 
knowledge, the state-of-the-art of DM-OEOs, and show that the performances of DM-OEOs are comparable to standard OEOs. Thus, direct modulation is a valuable alternative, which should be considered in particular when one aims at monolithic photonic chips [10]. The paper is organized as follows. In section II we present the experimental results of the single and dual loop DM-OEO, together with a comparison to a simple phase noise model. We offer some conclusions and perspectives in section III.

\section{EXPERIMENTAL RESULTS}

\section{II.1. Single loop DM-OEO}

The simplest possible OEO consists in a directlymodulated laser, controlled by a bias current and a modulation current (Fig. 1). Light from the laser passes through a km-long optical fiber before being detected by a photodiode. The generated photocurrent is then filtered by a high-Q dielectric resonant filter centered at $10 \mathrm{GHz}$, suitably amplified, and fed back into the laser modulation port. In the standard OEO [1], the MZM provides the optical modulation function. Thanks to its nonlinear response, the MZM also displays a power saturation behavior that sets the stable working point of the oscillator, fulfilling the Barkhausen condition $\left\|A \beta\left(f_{0}\right)\right\|=1$, where $A$ and $\beta\left(f_{0}\right)$ represent the gain and the transfer function of the feedback loop respectively [11]. In our scheme, it is the electrical amplifier that plays the role of the saturating component, stabilizing the circulating RF power.

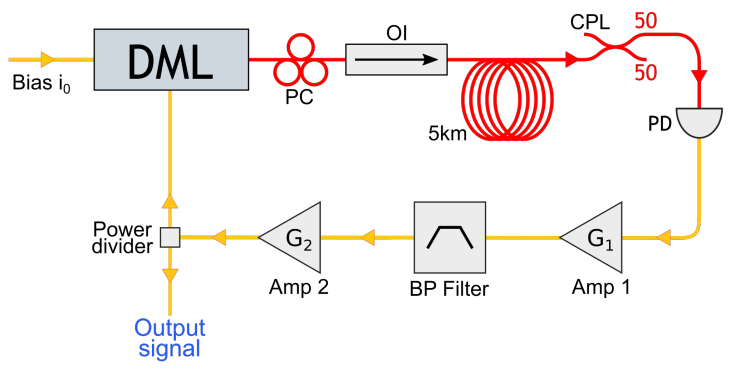

Figure 1: Single loop DM-OEO scheme. DML: Directly Modulated Laser, OI: Optical isolator, PC: Polarization controller CPL: Optical 50/50 coupler, PD: Photodiode.

Fig. 1 shows the experimental setup of the single loop DM-OEO architecture. A commercial telecommu- nication DFB laser (NEL, NLK5C5E2KA), with a modulation bandwidth larger than $10 \mathrm{GHz}$ and an overall efficiency $\eta_{L}=0.07 \mathrm{~W} / \mathrm{A}$ is used as the electrical to optical conversion component at $10 \mathrm{GHz}$. The optical delay is induced by a $5 \mathrm{~km}$-long SMF fiber, which is connected to a 50/50 coupler offering at the same time an optical output port to the DM-OEO and an adapted level of optical power on the photodiode. The photodiode is an InGaAs semiconductor PIN photo-diode (Nortel, PP-10G) with a responsivity $\eta_{P h . D}=0.88 \mathrm{~A} / \mathrm{W}$, a saturation power of $0 \mathrm{dBm}$ and a $500 \mathrm{~V} / \mathrm{A}$ transimpedance gain at its output. The output voltage is amplified by a $36 \mathrm{~dB}$ RF amplifier (Amp 1) with a +14 $\mathrm{dBm} \mathrm{P} 1 \mathrm{~dB}$ output power and a $0.7 \mathrm{~dB}$ typical noise figure. A custom high $Q$-factor $(Q=2500)$ dielectric filter from LAAS ${ }^{1}$ with a $-3 \mathrm{~dB}$ bandwidth of $4 \mathrm{MHz}$ and centered around $f_{0}=10.00485 \mathrm{GHz}$ is added at the output of Amp 1. Due to some degradation of its internal connectors, the filter displays insertion losses of about $10 \mathrm{~dB}$. The filtered signal is then amplified again by a $28 \mathrm{~dB}$ RF amplifier (Amp 2) which has a P1dB output power of $18 \mathrm{dBm}$ and a $8 \mathrm{~dB}$ typical noise figure. After Amp 2, the signal passes through a $3 \mathrm{~dB}$ power divider. Half of the RF power is fed back to the laser through the RF input port, the other half constitutes the useful output signal, and is directed towards a phase noise analyzer (Rohde \& Schwarz FSWP26). The laser is driven by a continuous bias current $i_{0}$, and controlled in temperature. In our experiment we set the bias current $i_{0}$ at $70 \mathrm{~mA}$ (the laser threshold is $i_{\text {th }}=10 \mathrm{~mA}$ ) and measure a mean optical power of $4.6 \mathrm{~mW}$ at the output of the laser and $1.35 \mathrm{~mW}$ at the input of the photodiode. An optical isolation stage was also added in front of the optical fiber in order to avoid optical feedback in the laser and the dynamical instabilities that it could cause.

Fig. 2 shows the RF spectrum of the output signal from the DM-OEO. An extracted electrical power of 15 $\mathrm{dBm}$ is available at the carrier frequency of 10.00436 $\mathrm{GHz}$. The adjacent peaks are non-oscillating resonant modes of the delay loop, apart from each other by the Free Spectral Range FSR $=\frac{c}{n L}=40 \mathrm{kHz}$, where $n$ and $L$ are the fiber refractive index and length respectively. The rejection ratio of the highest peaks is of $60 \mathrm{~dB}$.

The spectrum profile presented in Fig. 2 is stable in time, in the sense that no mode hops are observed.

\footnotetext{
${ }^{1}$ Laboratoire d'Analyse et d'Architecture des Systèmes F-31077 Toulouse, France
} 


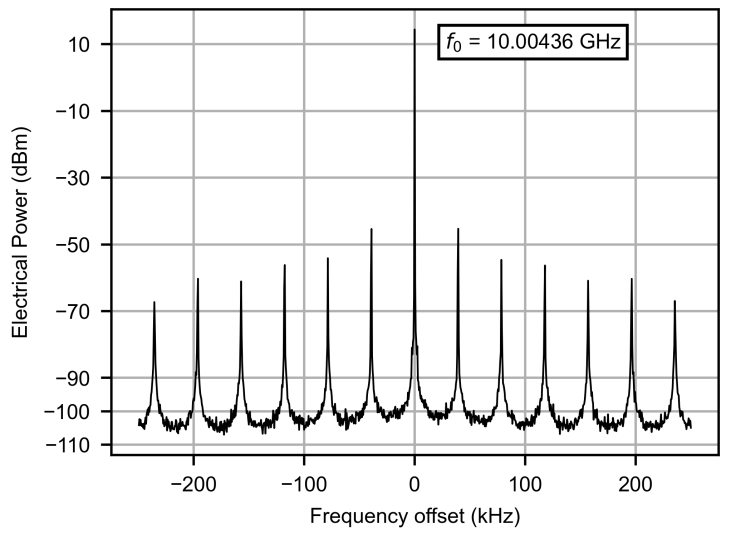

Figure 2: Electrical spectrum of the $10 \mathrm{GHz}$ carrier in the single loop DM-OEO. Span: $500 \mathrm{kHz}$. Resolution Bandwidth: $10 \mathrm{~Hz}$.

However, a slow constant drift of the carrier frequency is present. We quantified the frequency drift by configuring the ESA trace settings on Max Hold (Fig. 3). We observed a drift towards low frequencies of $1 \mathrm{kHz}$

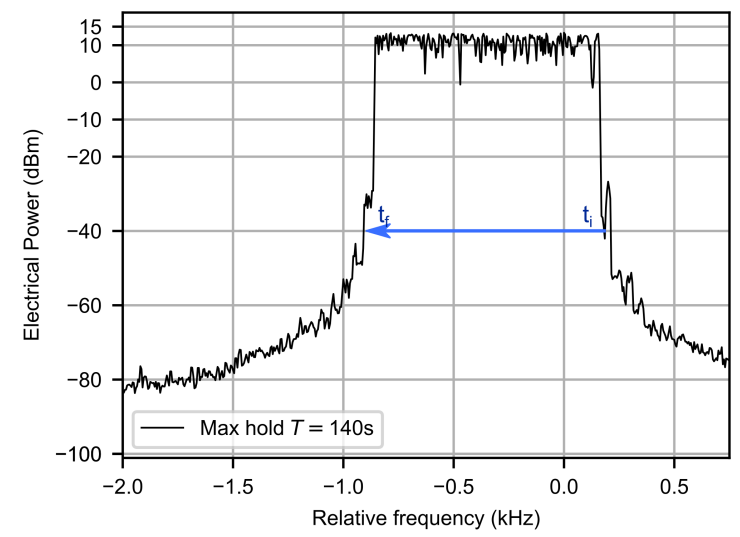

Figure 3: Max hold of the $10 \mathrm{GHz}$ carrier drift in the single loop DM-OEO. Resolution Bandwidth: $5 \mathrm{~Hz}$. Measurement time: 140s. Center frequency: $10.004355 \mathrm{GHz}$.

in $140 \mathrm{~s}$, corresponding to $7.2 \mathrm{~Hz} / \mathrm{s}$. This absolute frequency stability is obtained without any particular thermal or mechanical stabilization effort (the setup is inside a non-thermalized plexiglass box, on an optical table). One may expect that such a drift would eventually lead to a mode hop. However, a drift of the order of the FSR would require more than one hour, consistent with our observation of no mode hops over a 10 minutes time scale. Given the fact that the carrier frequency diminishes in time, the drift may be attributed to a slow thermal expansion of the fiber loop.

The distortions of the saturated second amplifier also generate harmonics at higher frequencies. Our ESA cannot analyze frequencies beyond $26.5 \mathrm{GHz}$ so we can only observe the second harmonic carrier (at 20 $\mathrm{GHz}$ ). Such a behavior has already been used to create a third harmonic generator in an OEO[12].

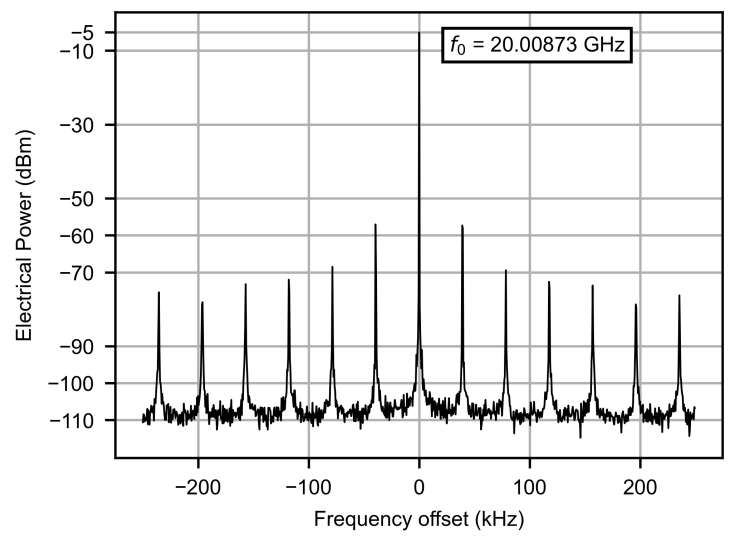

Figure 4: Electrical spectrum of the $20 \mathrm{GHz}$ carrier in the single loop DM-OEO. Span: $500 \mathrm{kHz}$. Resolution Bandwidth: $30 \mathrm{~Hz}$. The difference in resolution bandwidth with respect to Fig. 2 is inessential and does not impact the spectrum.

Fig. 4 shows the RF spectrum of the $2^{\text {nd }}$ harmonic, exhibiting an oscillation power of $-5 \mathrm{dBm}$ and a rejection ratio of $50 \mathrm{~dB}$ of the non-oscillating modes.

In Fig. 5 we present the phase noise spectra of both $10 \mathrm{GHz}$ and $20 \mathrm{GHz}$ signals, measured with Rohde \& Schwarz FSWP26 allowing the tracking of the oscillating frequency and measurement of precise phase noise spectra using cross correlation to lower the influence of its internal oscillator noise. Our experiment shows a phase noise of $-135 \mathrm{dBc} / \mathrm{Hz}$ at $10 \mathrm{kHz}$ offset from the $10 \mathrm{GHz}$ carrier and of $-127 \mathrm{dBc} / \mathrm{Hz}$ at $10 \mathrm{kHz}$ offset from the $20 \mathrm{GHz}$ carrier. The phase noise floors are of $-140 \mathrm{dBc} / \mathrm{Hz}$ at $10 \mathrm{GHz}$ and $-130 \mathrm{dBc} / \mathrm{Hz}$ at $20 \mathrm{GHz}$ respectively. The non-oscillating modes appear as spurs in the phase noise spectra. The highest ones are at 40 $\mathrm{kHz}$ from the carriers, and their noise level is between $-60 \mathrm{dBc} / \mathrm{Hz}$ and $-80 \mathrm{dBc} / \mathrm{Hz}$ (actually the spurs levels suggested by Fig. 5 are probably underestimated by about $10 \mathrm{~dB}$, see the discussion in II.3). For the sake of 


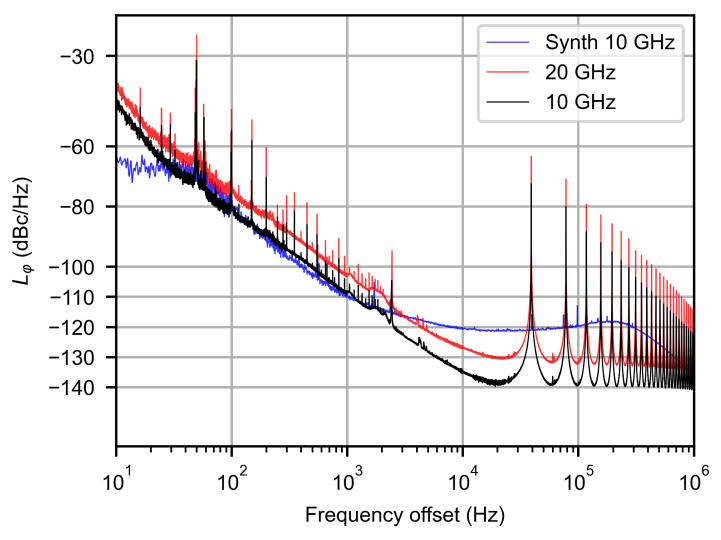

Figure 5: Phase noise spectrum of the $10 \mathrm{GHz} \mathcal{E} 20 \mathrm{GHz}$ carriers in the single loop DM-OEO. Synth: Phase noise of a low-noise Rohde E Schwarz SMF100A-B1 OCXO electrical synthesizer at $10 \mathrm{GHz}$. Measurements were made with a 50 XCORR factor (50 cross correlations at $10 \mathrm{~Hz}$ ) and $a .1 \% \mathrm{RBW}$.

comparison, we plotted the phase noise of a electrical low-noise synthesizer (Rohde \& Schwarz SMF100A-B1 OCXO) oscillating at $10 \mathrm{GHz}$. One can see that the $\mathrm{DM}-\mathrm{OEO}$ signal is as good as the synthesizer between $30 \mathrm{~Hz}$ and $2 \mathrm{kHz}$, and better than it after $2 \mathrm{kHz}$, apart from the spurs. For instance, at $10 \mathrm{kHz}$ from the carrier the OEO phase noise is $15 \mathrm{~dB}$ below the synthesizer.

\section{II.2. Dual loop DM-OEO}

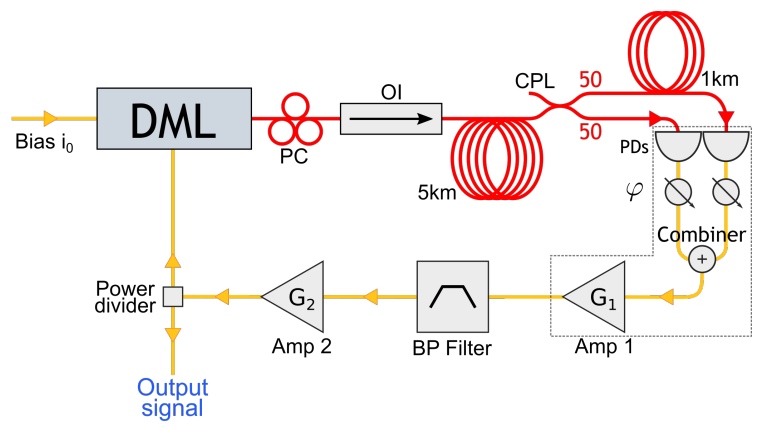

Figure 6: Dual loop DM-OEO schematic. DML: Directly Modulated Laser, PC: Polarization controller, OI: Optical isolator, CPL: Optical 50/50 coupler, PDs: Photo-diodes, $\varphi$ : Phase-shifters.

The single loop OEO architecture provides a high rejection ratio and low phase noise, but non-resonant spurs degrade the phase noise at the optical FSR frequencies and they can be very high $(-60 \mathrm{dBc} / \mathrm{Hz}$ or more). One way to lower them is to use a dual loop approach [13]. Such an approach is not specific to the DM-OEO, and has been widely studied with external modulation schemes. By splitting the laser output signal in two different paths with different delays and combining them electrically after the photodiode detection, we are able to filter out the spurs by RF interferometry.

Fig. 6 shows our experimental implementation of the dual loop DM-OEO. At variance with the single loop scheme, the second output port of the 50/50 coupler is connected to a $1 \mathrm{~km}$ fiber followed by a second identical PP-10G transimpedance photodiode. Then each of the two electrical signals passes through a variable phase shifter before being combined. The resulting signal is amplified by the $36 \mathrm{~dB}$ amplifier and the following link scheme is the same as that of the previous section. Our configuration corresponds to a dual loop architecture with $5 \mathrm{~km}$ and $6 \mathrm{~km}$ loop lengths. Only the modes that are resonant for both loops are not filtered out, and thus are modes of the overall oscillator. We optimized the phase noise by setting the bias current of the laser to $73 \mathrm{~mA}$. We then measured a laser optical power of $4.9 \mathrm{~mW}$, an optical power of $1.2 \mathrm{~mW}$ at the end of the $1 \mathrm{~km}$ fiber and $1.3 \mathrm{~mW}$ on the second output port of the coupler. Empirically we observed that the bias current, the laser modulation power, and the electrical phase-shifts are determinant in the optimization of the phase noise level. We also observed, consistently with [14], that the performance in terms of phase noise is improved by adjusting the optical power slightly beyond the saturation level of the photodiodes. Indeed, the P1dB input power of our detector is $1 \mathrm{~mW}$ but the mean optical power we inject is around $1.2 \mathrm{~mW}$.

The resulting electrical spectrum at $10 \mathrm{GHz}$ (Fig. 7) exhibits a high rejection ratio of more than $80 \mathrm{~dB}$. In particular, the closest-to-the-carrier spurs (at $40 \mathrm{kHz}$ ) are at $-90 \mathrm{dBm}$ for a carrier power of $13 \mathrm{dBm}$. As we use the second RF amplifier $(28 \mathrm{~dB})$ at its saturation point, we observe SHG at $20 \mathrm{GHz}$ as in the single loop architecture, with a relatively high $-7 \mathrm{dBm}$ power at $20 \mathrm{GHz}$, and a high rejection ratio of $90 \mathrm{~dB}$ near the carrier. The dual loop architecture exhibits better RF spectra profiles, while the signal powers remain of the 


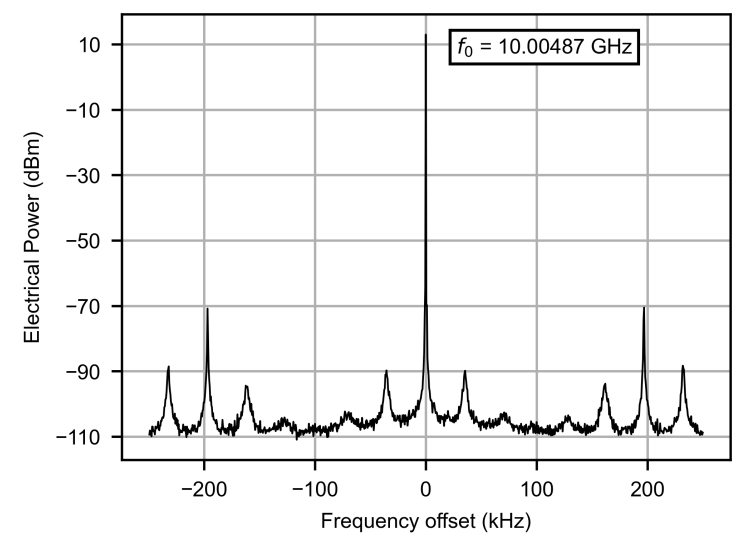

Figure 7: Electrical spectrum of the $10 \mathrm{GHz}$ carrier in the dual loop DM-OEO. Span: $500 \mathrm{kHz}$. Resolution Bandwidth: $5 \mathrm{~Hz}$.

same order both at 10 and $20 \mathrm{GHz}$.

Fig. 8 shows the phase noise of the two carriers in the dual loop setup. The phase noise floors are similar to those of the single loop architecture $(-140 \mathrm{dBc} / \mathrm{Hz}$ for the $10 \mathrm{GHz}$ carrier), but they can dive off by $5 \mathrm{~dB}$ at certain frequencies. For example, the $10 \mathrm{GHz}$ carrier phase noise floor can reach a value of $-145 \mathrm{dBc} / \mathrm{Hz}$. This indicates that the real floor should be at -145 $\mathrm{dBc} / \mathrm{Hz}$ but that the electrical interference quality (the contrast) is degrading the phase noise level.

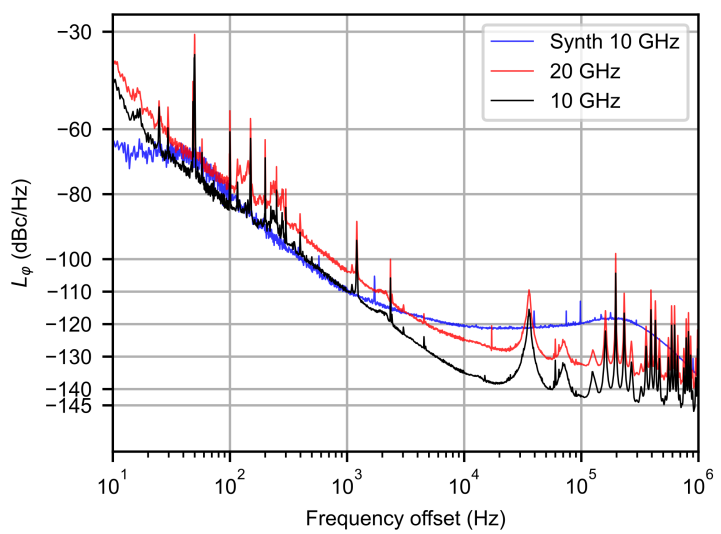

Figure 8: Phase noise spectrum of the $10 \mathrm{GHz} \mathcal{E} 20 \mathrm{GHz}$ carriers in the dual loop DM-OEO. Synth: Phase noise of a lownoise Rohde E Schwarz SMF100A-B1 OCXO electrical synthesizer at $10 \mathrm{GHz}$. Measurements were made with a 30 XCORR factor (30 cross correlations at $10 \mathrm{~Hz}$ ) and a $1 \%$ RBW.
The most notable phase noise difference is the level of the spurs that has been highly improved. The first spur of the $10 \mathrm{GHz}$ carrier has a phase noise of -115 $\mathrm{dBc} / \mathrm{Hz}$ at $40 \mathrm{kHz}$ offset. This is at least $43 \mathrm{~dB}$ less than in the single loop configuration. Moreover, because of the periodicity of the Vernier effect we still observe high spurs but less frequently. The highest spur is at $195 \mathrm{kHz}$ from the $10 \mathrm{GHz}$ carrier with a phase noise level of about $-100 \mathrm{dBc} / \mathrm{Hz}$. We used the same method than in the previous section to measure the drift of the $10 \mathrm{GHz}$ carrier. The dual loop DM-OEO shows a drift velocity of $9.2 \mathrm{~Hz} / \mathrm{s}$ towards low frequencies.

\section{II.3. Comparison with a phase noise model}

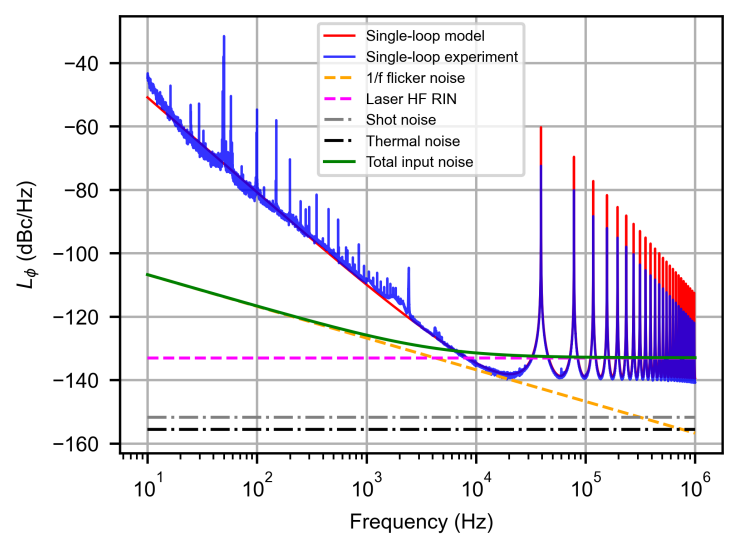

Figure 9: Comparison of the model eq. 1 with the experimental results. The input noises (thermal, shot, laser RIN, flicker) as well as the resulting total input noise are also shown.

In order to obtain some insights in the performances of our DM-OEO, we compared our experimental results with a simplified version of the model proposed by Lelièvre et al. [15], using their proposed eq. (3):

$$
S_{\phi_{o s c}}(f)=\left|\frac{\beta_{f}(f)}{1-\beta_{f}(f) \beta_{d}(f)}\right|^{2} S_{\psi_{1}}(f)
$$

where $S_{\phi_{\text {osc }}}(f)$ is the phase noise spectrum of the $\mathrm{OEO}, \beta_{f}(f)$ and $\beta_{d}(f)$ the microwave filter and the delay line transfer functions respectively, and $S_{\psi_{1}}(f)$ the input phase noise after the photodiode. 
We considered the following input noise sources: thermal and shot noise, laser relative intensity noise (RIN) at the oscillation frequency $f_{0}$, and a flicker noise source with spectral density of the form $b_{-1} f^{-1}$. So the input phase noise $S_{\psi_{1}}(f)$ reads

$$
S_{\psi_{1}}(f)=S_{\text {th }}+S_{\text {shot }}+S_{R I N_{H F}}+S_{\text {flicker }}
$$

The laser RIN at $10 \mathrm{GHz}$ was experimentally measured to be $-137 \mathrm{~dB} / \mathrm{Hz}$. We calculated the corresponding input phase noise using Lelièvre's formulas (12) et (13), with a modulation depth $m=0.9$, and injected it in eq. 1. As Fig. 9 shows, there is a very good agreement with the experimental noise floor (note that the minima of the phase noise of the oscillator are $6 \mathrm{~dB}$ below the in-loop noise level, an effect discussed in [11], p. 139). So we can conclude that, for frequencies above $10 \mathrm{kHz}$, the laser RIN at high frequency is the factor limiting the performances of our system. In order to reproduce the experimental noise spectrum for frequencies below $10 \mathrm{kHz}$, it is necessary to include an additional noise source $S_{\text {flicker }}=b_{-1} f^{-1}$ with $b_{-1}=2.110^{-10}$. This additional flicker noise allows obtaining a good agreement with experiments, and is most probably due to the RF amplifiers and to the conversion of laser frequency noise (to which frequency chirping caused by direct modulation may also contribute) into RF phase noise via the fiber dispersion. At present the data for these two contributions are not available to us, and we cannot say which of the two effects is the most important. In fig. 9, the level of the spurs is higher in the model than in the experimental curve. This is probably not a limitation of the model, but an artifact due to the insufficient frequency resolution of the phase noise measurement, as in [15]. This interpretation is corroborated by Fig. 10, where the prediction of the model for the dual-loop configuration [15] is compared with the experimental results. In this case the first spur is considerably lowered and broadened, and it can be seen that its height is correctly predicted by the model. This suggests that the model predictions for the spurs levels are correct, and thus that the real level of the spurs in the single-loop configuration is around $10 \mathrm{~dB}$ higher than what is suggested by Fig. 5 .

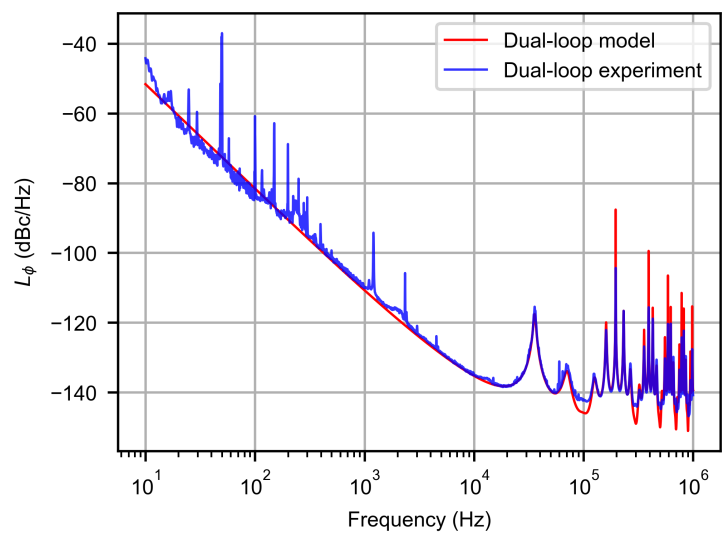

Figure 10: Comparison of the model for the dual-loop configuration with the experimental results. The input noises are the same as in Fig. 9.

\section{CONCLUSION AND PERSPECTIVES}

The following table summarizes the performances of the different configurations of our system.

Table 1: Phase Noise Comparisons

\begin{tabular}{l|c|c|c|c|c|} 
& Synth $10 \mathrm{GHz}$ & SL $10 \mathrm{GHz}$ & SL $20 \mathrm{GHz}$ & DL $10 \mathrm{GHz}$ & DL 20 GHz \\
\hline P.N. at $10 \mathrm{KHz}(\mathrm{dBc} / \mathrm{Hz})$ & -120 & -135 & -127 & -135 & -125 \\
\hline P.N. floor $(\mathrm{dBc} / \mathrm{Hz})$ & & -140 & -132 & -140 & -132 \\
\hline First spur level $(\mathrm{dBc} / \mathrm{Hz})$ & & $>-72$ & $>-63$ & -115 & -110 \\
\hline E. Power $(\mathrm{dBm})$ & 15 & 15 & -5 & 13 & -7 \\
\hline
\end{tabular}

We can compare these figures to some published results. Wishon et al.[6] shows typical phase noise levels of $-107 \mathrm{dBc} / \mathrm{Hz}$, while displaying the ability to tune the carrier frequency. Sung et al.[5] reports a $20 \mathrm{GHz}$ carrier with phase noise of $-123 \mathrm{dBc} / \mathrm{Hz}$, using a setup that involves strong injection locking to a master laser. Qi et al.[9], using a directly-modulated home-made $1.3 \mu \mathrm{m}$ laser, obtained a phase noise of $-125 \mathrm{dBc} / \mathrm{Hz}$ at $10 \mathrm{kHz}$ from the carrier. Considering that the noise floor in [9] is similar to ours, we can ascribe most of the $10 \mathrm{~dB}$ phase noise improvement at $10 \mathrm{kHz}$ to the longer resonator length in our setup, as is confirmed by simulations using eq. 1 . Concerning standard OEOs with external modulation, phase noises as low as $-145 \mathrm{dBc} / \mathrm{Hz}$ [15] can be obtained at the cost of a careful optimization of all elements as well as the use of high power lasers (for instance, in [15] a DFB 
laser delivering $120 \mathrm{~mW}$ was used). The lowest noise reported to date is, to the best of our knowledge, -163 $\mathrm{dBc} / \mathrm{Hz}$ at $6 \mathrm{kHz}$ offset [14], in a setup using a high power solid-state $\mathrm{Nd}$ :YAG laser and a $16 \mathrm{~km}$ optical fiber.

In conclusion, we demonstrated the possibility of a stable and powerful oscillation in a DM-OEO both in the single loop and dual loop architectures. We measured a $15 \mathrm{dBm}$ output RF power and a phase noise level of $-135 \mathrm{dBc} / \mathrm{Hz}$ at $10 \mathrm{kHz}$ from a $10 \mathrm{GHz}$ carrier in both configurations. In the dual-loop configuration, the first spur was as low as $-115 \mathrm{dBc} / \mathrm{Hz}$. The system also generates a low-phase noise $20 \mathrm{GHz}$ carrier. The phase noise analysis showed that the laser RIN is the limiting component of the system for frequencies higher than $10 \mathrm{kHz}$. For better performance one should choose a less noisy laser, which could lead in principle, all other parameters being equal, to an improvement of about $15 \mathrm{~dB}$ of the noise floor, for shotnoise-limited operation. In practice the best strategy to improve the performances would be to use a more powerful laser as in [15]. Apart from decreasing the laser RIN, that would also have the added benefit of reducing the need for electrical amplification. With a lower noise floor, a reduction of the fiber loop length may also be acceptable. These results are, to the best of our knowledge, the state-of-the-art of DM-OEOs. This more compact architecture avoiding external modulation is particularly interesting in the perspective of monolithic integration of OEOs in photonic integrated circuits [10]. In this respect, the integration of the delay function is probably the most challenging part. Integrated $\mathrm{Si}_{3} \mathrm{~N}_{4}$ resonators [16] are promising devices for such purpose.

\section{ACKNOWLEDGMENT}

We thank O. Llopis from LAAS for providing us with the RF filter, and A. Carré, G. Loas, C. Peucheret and F. Bondu for fruitful discussions.

\section{REFERENCES}

[1] X. S. Yao and L. Maleki, "Optoelectronic microwave oscillator," Journal of the Optical Society of America B, vol. 13, no. 8, p. 1725, aug 1996.
[2] Y. K. Chembo, D. Brunner, M. Jacquot, and L. Larger, "Optoelectronic oscillators with timedelayed feedback," Reviews of Modern Physics, vol. 91, no. 3, p. 35006, 2019.

[3] G. R. Goune Chengui, P. Woafo, and Y. K. Chembo, "The Simplest Laser-Based Optoelectronic Oscillator: An Experimental and Theoretical Study," Journal of Lightwave Technology, vol. 34, no. 3, pp. 873-878, 2016.

[4] G. Giacomelli, M. Calzavara, and F. T. Arecchi, "Instabilities in a semiconductor laser with delayed optoelectronic feedback," Optics Communications, vol. 74, no. 1-2, pp. 97-101, dec 1989.

[5] H. K. Sung, X. Zhao, E. K. Lau, D. Parekh, C. J. Chang-Hasnain, and M. C. Wu, "Optoelectronic oscillators using direct-modulated semiconductor lasers under strong optical injection," IEEE Journal on Selected Topics in Quantum Electronics, vol. 15, no. 3, pp. 572-577, 2009.

[6] M. J. Wishon, D. Choi, T. Niebur, N. Webster, Y. K. Chembo, E. A. Viktorov, D. S. Citrin, and A. Locquet, "Low-noise $x$-band tunable microwave generator based on a semiconductor laser with feedback," IEEE Photonics Technology Letters, vol. 30, no. 18, pp. 1597-1600, 2018.

[7] A. Thorette, M. Romanelli, S. Bouhier, F. Van Dijk, M. Vallet, and M. Alouini, "Hybrid opto-electronic oscillator for single-sideband microwave photonics," Electronics Letters, vol. 54, no. 11, pp. 706-708, may 2018.

[8] M. L. Liao, J. L. Xiao, Y. Z. Huang, H. Z. Weng, J. Y. Han, Z. X. Xiao, and Y. D. Yang, "Tunable Optoelectronic Oscillator Using a Directly Modulated Microsquare Laser," IEEE Photonics Technology Letters, vol. 30, no. 13, pp. 1242-1245, jul 2018.

[9] B. Qi, H. Wang, B. Zhang, L. Xie, and P. Gong, "Improvement of the phase noise model based on an optoelectronic oscillator using a directly modulated distributed feedback laser," Optics Communications, vol. 488, p. 126848, jun 2021.

[10] P. Primiani, H. Débrégéas, D. Lanteri, M. Alouini, and F. Van Dijk, "Electro-Absorption modulatorbased optoelectronic oscillator," in MWP 2017 - 
2017 International Topical Meeting on Microwave Photonics, vol. 2017-December. Institute of Electrical and Electronics Engineers Inc., dec 2017, pp. 1-3.

[11] E. Rubiola, Phase noise and frequency stability in oscillators. Cambridge University Press, jan 2008, vol. 9780521886 .

[12] W. Xu, C. Yang, Z. Wang, and W. Zhao, "Frequency-tripling OEO based on frequency multiplication in saturated electronic amplifier," Optik, vol. 194, oct 2019.

[13] X. S. Yao, L. Maleki, Y. Ji, G. Lutes, and M. Tu, "Dual-loop opto-electronic oscillator," in Proceedings of the Annual IEEE International Frequency Control Symposium. IEEE, 1998, pp. 545-549.

[14] D. Eliyahu, D. Seidel, and L. Maleki, “RF amplitude and phase-noise reduction of an optical link and an opto-electronic oscillator," IEEE Transactions on Microwave Theory and Techniques, vol. 56, no. 2, pp. 449-456, 2008.

[15] O. Lelievre, V. Crozatier, P. Berger, G. Baili, O. Llopis, D. Dolfi, P. Nouchi, F. Goldfarb, F. Bretenaker, L. Morvan, and G. Pillet, "A model for designing ultralow noise single- and dual-loop 10GHz optoelectronic oscillators," Journal of Lightwave Technology, vol. 35, no. 20, pp. 4366-4374, 2017.

[16] D. T. Spencer, J. F. Bauters, M. J. Heck, and J. E. Bowers, "Integrated waveguide coupled $s i_{3} n_{4}$ resonators in the ultrahigh-q regime," Optica, vol. 1, no. 3, pp. 153-157, 2014. 